\title{
Supplementary Material for Adverse Selection, Lemons Shocks and Business Cycles*
}

\author{
Daisuke Ikeda $^{\dagger}$
}

April 2019

\begin{abstract}
This material presents the derivation of a solution to the optimal contracting problem in the partial equilibrium model (the proof of Proposition 1 in the paper), the proof of Proposition 2, the system of equations for the dynamic general equilibrium model, the calculation of the steady state, the derivation of a key equation regarding lemons shocks, and a version of the model with an exogenous countercyclical wage markup. Moreover, it presents a model with risk shocks and a version of the model presented in the paper with a shock to lenders' perception about borrowers' riskiness.
\end{abstract}

\section{A. Optimal Contracting Problem}

This section first provides the derivation of a solution to the optimal contracting problem of the model with asymmetric information, presented in Section 2.3 in the paper. By doing so it provides the proof of Proposition 1. Next, this section presents the proof of Proposition 2, which shows the results of the comparative statics analysis of the model. The notations used in this material are the same as those in the paper, otherwise they would be noted.

\footnotetext{
${ }^{*}$ The views expressed in this material are solely the responsibility of the author and should not be interpreted as reflecting views of the Bank of Japan, Federal Reserve Bank of Dallas or the Federal Reserve System.

†Bank of Japan, 2-1-1 Nihonbashi-Hongokucho,Chuo-ku,Tokyo 103-8660 Japan. E-mail: daisuke.ikeda@boj.or.jp.

†Supplement DOI: https://doi.org/10.24149/gwp361supp
} 


\section{A1. Proof of Proposition 1}

The optimal contracting problem for the model with asymmetric information is reproduced here for convenience. In the problem, the type- $(n, p)$ entrepreneur chooses a bank that offers the schedule of contracts, $\left\{B_{n}(p), X_{n}(p)\right\}_{p=\underline{p}}^{\bar{p}}$, that maximizes the expected profits,

$$
\max _{\left\{B_{n}(p), X_{n}(p)\right\}_{p=\underline{p}}^{\bar{p}}} \int_{\underline{p}}^{\bar{p}}\left[R^{k}\left(N_{n}+B_{n}(p)\right)-p X_{n}(p)\right] d F(p),
$$

subject to the bank zero profit condition,

$$
\int_{\underline{p}}^{\bar{p}}\left[p X_{n}(p)-R B_{n}(p)\right] d F(p)=0,
$$

the entrepreneur's participation constraint,

$$
p \theta(p) R^{k} B_{n}(p)-p X_{n}(p) \geq 0, \quad \forall p \in[\underline{p}, \bar{p}]
$$

the entrepreneur's pledgeability constraint,

$$
p X_{n}(p) \leq \phi p \theta(p) R^{k}\left[N_{n}+B_{n}(p)\right], \quad \forall p \in[\underline{p}, \bar{p}]
$$

and the entrepreneur's incentive constraint,

$$
R^{k} B_{n}(p)-p X_{n}(p) \geq R^{k} B_{n}(\tilde{p})-p X_{n}(\tilde{p}), \quad \forall p, \tilde{p} \in[\underline{p}, \bar{p}]
$$

By assumption $p \theta(p)=1$ so that the expected return is identical at $R^{k}$ for all entrepreneurs.

This problem can be solved in four steps.

Step 1: Suppose that there exists a threshold $p^{*}$ such that entrepreneurs with riskiness $p>p^{*}$ do not get a loan, which will be verified later. Then the incentive constraint (A5) implies that the expected profit from borrowing for the type- $(n, p)$ entrepreneur is given by:

$$
\begin{array}{ll}
\text { if } p>p^{*} & R^{k} B_{n}(p)-p X_{n}(p)=-p X_{n}(p) \leq 0, \\
\text { if } p=p^{*} & R^{k} B_{n}(p)-p X_{n}(p)=0 \\
\text { if } p<p^{*} & R^{k} B_{n}(p)-p X_{n}(p)>0 .
\end{array}
$$


The equality in the first equation follows from the presumption that $B_{n}(p)=0$ for $p>p^{*}$. From the participation constraint $(\mathrm{A} 3), X_{n}(p)=0$ for entrepreneurs with $p>p^{*}$. For entrepreneurs with $p \leq p^{*}$ the participation constraint is satisfied, which is straightforward from the expected profit of borrowing (A6). Now the original problem is written as:

$$
\max _{\left\{p^{*},\left\{X_{n}(p), B_{n}(p)\right\}_{p=\underline{p}}^{p^{*}}\right\}} \int_{\underline{p}}^{p^{*}}\left[R^{k} B_{n}(p)-p X_{n}(p)\right] d F(p),
$$

subject to the zero profit condition (A2), the pledgeability constraint (A4), and the incentive constraint (A5). A constant term, which is irrelevant to the problem, is omitted from (A7).

Step 2: Let $\Pi_{n}(\tilde{p} ; p) \equiv R^{k} B_{n}(\tilde{p})-p X_{n}(\tilde{p})$ denote the expected profits for the type$(n, p)$ entrepreneur who has chosen the pair $\left\{B_{n}(\tilde{p}), X_{n}(\tilde{p})\right\}$. The incentive constraint (A5) requires that $\Pi_{n}(\tilde{p} ; p)$ is maximized at $\tilde{p}=p$. As in a standard mechanism design problem, the incentive constraint can be replaced with: $\forall p \in\left[\underline{p}, p^{*}\right]$,

$$
\begin{aligned}
& R^{k}\left[d B_{n}(p) / d p\right]-p\left[d X_{n}(p) / d p\right]=0 \\
& d X_{n}(p) / d p \leq 0
\end{aligned}
$$

The local incentive compatibility constraint (A8) corresponds to the first-order condition of the problem of maximizing $\Pi_{n}(\tilde{p} ; p)$ with respect to $\tilde{p}$, with $\tilde{p}$ evaluated at $\tilde{p}=p$, and the monotonicity constraint (A9) corresponds to its second-order condition. Let $\Pi_{n}^{*}(p)$ define $\Pi_{n}^{*}(p) \equiv \Pi_{n}(p ; p)$. Applying the envelop theorem to $\Pi_{n}^{*}(p)$ yields $d \Pi_{n}^{*}(p) / d p=$ $\left[d B_{n}(p) / d p\right]-p\left[d X_{n}(p) / d p\right]-X_{n}(p)=-X_{n}(p)$, where the local incentive compatibility constraint (A8) is used in the first equality. Reminding that $\Pi_{n}^{*}\left(p^{*}\right)=0$ from (A6), integrating $d \Pi_{n}^{*}(x) / d x$ over the interval $\left[p, p^{*}\right]$ yields:

$$
\Pi_{n}^{*}(p)=R^{k} B_{n}(p)-p X_{n}(p)=\int_{p}^{p^{*}} X_{n}(x) d x
$$

Now define $\mathbf{X}_{n}(p)$ as $\mathbf{X}_{n}(p) \equiv \int_{\underline{p}}^{p} X_{n}(x) d x$. Then, using (A10), the expected profits of the 
type- $n$ entrepreneur before $p$ is realized, (A7), is given by:

$$
\begin{aligned}
\int_{\underline{p}}^{p^{*}}\left[R^{k} B_{n}(p)-p X_{n}(p)\right] d F(p) & =\int_{\underline{p}}^{p^{*}} \int_{p}^{p^{*}} X_{n}(x) d x d F(p) \\
& =\int_{\underline{p}}^{p^{*}}\left[\mathbf{X}_{n}\left(p^{*}\right)-\mathbf{X}_{n}(p)\right] f(p) d p \\
& =\mathbf{X}_{n}\left(p^{*}\right) F\left(p^{*}\right)-\int_{\underline{p}}^{p^{*}} \mathbf{X}_{n}(p) f(p) d p
\end{aligned}
$$

where $F(\underline{p})=0$ was used in the final equality. Note that:

$$
\frac{d}{d p}\left[\mathbf{X}_{n}(p) F(p)\right]=X_{n}(p) F(p)+\mathbf{X}_{n}(p) f(p) .
$$

Combining the two equations above yields:

$$
\begin{aligned}
& \int_{\underline{p}}^{p^{*}}\left[R^{k} B_{n}(p)-p X_{n}(p)\right] d F(p) \\
= & \mathbf{X}_{n}\left(p^{*}\right) F\left(p^{*}\right)-\int_{\underline{p}}^{p^{*}}\left\{\frac{p}{d p}\left[\mathbf{X}_{n}(p) F(p)\right]-X_{n}(p) F(p)\right\} d p \\
= & \mathbf{X}_{n}\left(p^{*}\right) F\left(p^{*}\right)-\left[\mathbf{X}_{n}\left(p^{*}\right) F\left(p^{*}\right)-\mathbf{X}_{n}(\underline{p}) F(\underline{p})\right]+\int_{\underline{p}}^{p^{*}} X_{n}(p) F(p) d p, \\
= & \int_{\underline{p}}^{p^{*}} X_{n}(p) F(p) d p .
\end{aligned}
$$

From (A10), the loan schedule $B_{n}(p)$ is given by:

$$
B_{n}(p)=\left[p X_{n}(p)+\int_{p}^{p^{*}} X_{n}(x) d x\right] / R^{k} .
$$

The local incentive compatibility constraint (A8) is satisfied as long as equation (A13) holds. By using equation (A12), (A13) and $B_{n}(p)=X_{n}(p)=0$ for $p>p^{*}$, the zero profit condition (A2) is written as:

$$
\int_{\underline{p}}^{p^{*}} \omega(p) X_{n}(p) d p=0
$$

where

$$
\omega(p) \equiv p f(p)-\frac{R}{R^{k}} p f(p)-\frac{R}{R^{k}} F(p) .
$$


Also, by using equation (A13), the pledgeabiliy constraint (A4) is written as:

$$
X_{n}(p) \leq \frac{\phi}{(1-\phi) p} R^{k} N_{n}+\frac{\phi}{(1-\phi) p} \int_{p}^{p^{*}} X_{n}(x) d x
$$

To summarize the result in Step 2, the entrepreneur's problem is now written as follows:

$$
\max _{\left\{p^{*},\left\{X_{n}(p)\right\}_{p=\underline{p}}^{p^{*}}\right\}} \int_{\underline{p}}^{p^{*}} X_{n}(p) F(p) d p
$$

subject to the monotonicity constraint (A9), the zero profit condition (A14), and the pledgeability constraint (A15) .

Step 3: Let $\lambda \geq 0$ and $\xi(p) \geq 0$ denote Lagrange multipliers associated with the zero profit condition (A14) and the pledgeability constraint (A15), respectively. The monotonicity constraint (A9) is assumed to hold and will be verified later. Then, the entrepreneur's problem is formulated in a Lagrangean form as:

$$
\begin{aligned}
\mathcal{L} & =\max _{\left\{p^{*},\left\{X_{n}(p)\right\}_{p=\underline{p}}^{p^{*}}\right\}} \int_{\underline{p}}^{p^{*}}\left\{F(p) X_{n}(p)+\lambda \omega(p) X_{n}(p)\right. \\
& \left.+\xi(p)\left[\frac{\phi}{1-\phi} R^{k} N_{n}+\frac{\phi}{1-\phi} \int_{p}^{p^{*}} X_{n}(x) d x-p X_{n}(p)\right]\right\} d p .
\end{aligned}
$$

Let $\Xi(p)$ define $\Xi(p)=\int_{\underline{p}}^{p} \xi(x) d x$. Then, the term associated with $\int_{p}^{p^{*}} X_{n}(x) d x$ in the Lagrangean is expanded as:

$$
\int_{\underline{p}}^{p^{*}} \xi(p) \int_{p}^{p^{*}} X_{n}(x) d x d p=\int_{\underline{p}}^{p^{*}} \xi(p)\left[\mathbf{X}_{n}\left(p^{*}\right)-\mathbf{X}_{n}(p)\right] d p=\Xi\left(p^{*}\right) \mathbf{X}_{n}\left(p^{*}\right)-\int_{\underline{p}}^{p^{*}} \xi(p) \mathbf{X}_{n}(p) d p
$$

Note that:

$$
\frac{d}{d p}\left[\Xi(p) \mathbf{X}_{n}(p)\right]=\xi(p) \mathbf{X}_{n}(p)+\Xi(p) X_{n}(p)
$$


Combining the two equations above yields:

$$
\begin{aligned}
\int_{\underline{p}}^{p^{*}} \xi(p) \int_{p}^{p^{*}} X_{n}(x) d x d p & =\Xi\left(p^{*}\right) \mathbf{X}_{n}\left(p^{*}\right)-\int_{\underline{p}}^{p^{*}}\left\{\frac{d}{d p}\left[\Xi(p) \mathbf{X}_{n}(p)\right]-\Xi(p) X_{n}(p)\right\} d p \\
& =\Xi\left(p^{*}\right) \mathbf{X}_{n}(p)-\left[\Xi\left(p^{*}\right) \mathbf{X}_{n}\left(p^{*}\right)-\int_{\underline{p}}^{p^{*}} \Xi(p) X_{n}(p) d p\right] \\
& =\int_{\underline{p}}^{p^{*}} \Xi(p) X_{n}(p) d p .
\end{aligned}
$$

Substituting this into the Lagrangean, the problem is written as follows:

$$
\begin{aligned}
\mathcal{L} & =\max _{\left\{p^{*},\left\{X_{n}(p)\right\}_{p=\underline{p}}^{p^{*}}\right\}} \int_{\underline{p}}^{p^{*}}\left\{F(p) X_{n}(p)+\lambda \omega(p) X_{n}(p)\right. \\
& \left.+\xi(p) \frac{\phi}{1-\phi} R^{k} N_{n}+\frac{\phi}{1-\phi} \Xi(p) X_{n}(p)-p \xi(p) X_{n}(p)\right\} d p .
\end{aligned}
$$

The maximization of $\mathcal{L}$ with respect to the payment schedule $\left\{X_{n}(p)\right\}_{p=\underline{p}}^{p^{*}}$ requires that the term under the integral be maximized with respect to $X_{n}(p)$ for all $p \in\left[\underline{p}, p^{*}\right]$. The first-order conditions with respect to $X_{n}(p)$ and $p^{*}$ are given, respectively, by:

$$
\begin{aligned}
& F(p)+\lambda \omega(p)+\frac{\phi}{1-\phi} \Xi(p)-p \xi(p)=0, \\
& \xi\left(p^{*}\right)=0
\end{aligned}
$$

Given that zero profit condition (A14) is binding so that $\lambda>0$, equation (A16) constitutes an integral equation for $\xi(p)$ with an initial condition, $\xi(\underline{p})=\lambda \omega(\underline{p}) / \underline{p}>0$. The solution to (A16) is given by:

$$
\xi(p)=\frac{F(p)+\lambda \omega(p)}{p}+\frac{\phi}{(1-\phi) p} p^{\frac{\phi}{1-\phi}} \int_{\underline{p}}^{p}[F(x)+\lambda \omega(x)]\left(\frac{1}{x}\right)^{\frac{1}{1-\phi}} d x
$$

Substituting (A18) into (A17) yields:

$$
\xi\left(p^{*}\right)=\frac{F\left(p^{*}\right)+\lambda \omega\left(p^{*}\right)}{p^{*}}+\frac{\phi}{(1-\phi) p^{*}}\left(p^{*}\right)^{\frac{\phi}{1-\phi}} \int_{\underline{p}}^{p^{*}}[F(x)+\lambda \omega(x)]\left(\frac{1}{x}\right)^{\frac{1}{1-\phi}} d x=0 .
$$

Uniform distribution: In the case of the uniform distribution, $F(p)=(p-\underline{p}) /(\bar{p}-\underline{p})$, 
the numerator of the first term in equation (A18) is given by:

$$
\begin{aligned}
F(p)+\lambda \omega(p) & =\lambda\left[p f(p)-\frac{R}{R^{k}} p f(p)\right]+\left(1-\lambda \frac{R}{R^{k}}\right) F(p) \\
& =\frac{1}{\bar{p}-\underline{p}}\left[\underline{p}\left(\lambda \frac{R}{R^{k}}-1\right)-p\left(2 \lambda \frac{R}{R^{k}}-1-\lambda\right)\right]
\end{aligned}
$$

Suppose contrarily $\lambda\left(R / R^{k}\right)-1<0$. Then, $F(p)+\lambda \omega(p)$ is increasing in $p$, which contradicts with condition (A17). Hence, it must be $\lambda\left(R / R^{k}\right)-1>0$, so that $F(p)+\lambda \omega(p)$ is decreasing in $p$. Because $F(p)+\lambda \omega(p)$ is decreasing and $F(\underline{p})+\lambda \omega(\underline{p})>0$, equation (A18) implies that $\xi(p)>0$ at least for $p \leq p_{0}$ where $F\left(p_{0}\right)+\lambda \omega\left(p_{0}\right)=0$. For $p>p_{0}, \xi(p)$ becomes decreasing in $p$. Equation (A19) implies that $\xi(p)$ then crosses the zero line at $p=p^{*}$. Therefore, under the assumption of the uniform distribution, $\xi(p)>0$ for $p<p^{*}$, i.e. the pledgeability constraint (A15) holds with equality for entrepreneurs with $p<p^{*}$.

Step 4: Assume that pledgeability constraint (A15) holds with equality for all $p<p^{*}$, which will be verified later. As shown in Step 3, the assumption holds under the uniform distribution. For $p=p^{*}$ the pledgeability constraint also holds with equality because of the continuity of the Lagrange multiplier $\xi(p)$. Then, solving the pledgeability constraint (A15) for $X_{n}(p)$ yields:

$$
X_{n}(p)=\left[\frac{\phi R^{k}}{1-\phi}\left(p^{*}\right)^{\frac{\phi}{1-\phi}}\right]\left(\frac{1}{p}\right)^{\frac{1}{1-\phi}} N_{n}
$$

Payment schedule $X_{n}(p)$ is strictly decreasing in $p$ so that it satisfies monotonicity constraint (A9). Substituting (A20) into zero profit condition (A14) yields:

$$
\int_{\underline{p}}^{p^{*}} \omega(p)\left(\frac{1}{p}\right)^{\frac{1}{1-\phi}} d p=0
$$

Under the assumption that (A21) has a unique solution for $p^{*}<\bar{p}$, the guess made in Step 1 is confirmed. With $p^{*}$ to hand, $\lambda$ is obtained from (A19) as:

$$
\lambda=\frac{F\left(p^{*}\right)+\frac{\phi}{1-\phi}\left(p^{*}\right)^{\frac{\phi}{1-\phi}} \int_{\underline{p}}^{p^{*}} F(x)\left(\frac{1}{x}\right)^{\frac{1}{1-\phi}} d x}{-\omega\left(p^{*}\right)}>0 .
$$

The inequality holds because $\omega\left(p^{*}\right)<0$ and $\omega(\underline{p})>0$, otherwise condition (A21) would not hold with unique $p^{*}$. The positive $\lambda$ confirms that the zero profit condition (A14) is 
binding. In addition, with $\lambda$ to hand, the binding pledgeability constraint, $\xi(p)>0$, for all $p<p^{*}$ can be verified from equation (A18). Finally, substituting (A20) into (A13) yields loan schedule $B_{n}(p)$ as:

$$
B_{n}(p)=\left[\frac{1}{1-\phi}\left(\frac{p^{*}}{p}\right)^{\frac{\phi}{1-\phi}}-1\right] N_{n}
$$

To summarize, the schedules of payment and loan are given by (A20) and (A23), respectively with $p^{*}$ given by (A21) for $p \in\left[\underline{p}, p^{*}\right]$, and are given by $X_{n}(p)=B_{n}(p)=0$ for $p \in\left(p^{*}, \bar{p}\right]$.

Regarding the derivation of the aggregate loan, aggregating (A23) over $p$ and $n$ yields:

$$
B=\left[\frac{1}{1-\phi} \int_{\underline{p}}^{p^{*}}\left(\frac{p^{*}}{p}\right)^{\frac{\phi}{1-\phi}} d F(p)-F\left(p^{*}\right)\right] N .
$$

Also, substituting (A20) into the zero profit condition (A2) and aggregating over $n$ yield:

$$
\frac{\phi R^{k}}{1-\phi}\left[\int_{\underline{p}}^{\bar{p}}\left(\frac{p^{*}}{p}\right)^{\frac{\phi}{1-\phi}} d F(p)\right] N-R B=0 .
$$

Combining (A24) and (A25) leads to:

$$
B=\frac{\phi\left(R^{k} / R\right)}{1-\phi\left(R^{k} / R\right)} F\left(p^{*}\right) N
$$

Uniform distribution: Under the assumption of the uniform distribution, $F(p)=(p-$ $\underline{p}) /(\bar{p}-\underline{p}), \omega(p)$ is strictly decreasing in $p$ if $R^{k} / R<2$ and $\omega(\underline{p})>0$. Hence, given that a solution to equation (A21) exists, a sufficient condition for its uniqueness is $R^{k} / R<2$. For existence, its sufficient condition is $\int_{\underline{p}}^{\bar{p}} \omega(p)(1 / p)^{\frac{1}{1-\phi}} d p<0$, which is written as:

$$
\frac{R^{k}}{R}<2-\underline{p} \frac{1-2 \phi}{\phi} \frac{\underline{p}^{\frac{-\phi}{1-\phi}}-\bar{p}^{\frac{-\phi}{1-\phi}}}{\bar{p}^{\frac{1-2 \phi}{1-\phi}}-\underline{p}^{\frac{1-2 \phi}{1-\phi}}}
$$

In the case of $\phi=1 / 2$, the right-hand-side of the inequality (A27) is reduced to $2-(\underline{p} / \bar{p})$. To summarize, in the case of the uniform distribution over interval $[\underline{p}, \bar{p}]$, a sufficient condition for the existence and uniqueness of a solution to (A21) is condition (A27). 


\section{A2. Proof of Proposition 2}

First, regarding comparative statics with respect to $R^{k} / R$, the threshold $p^{*}$ is determined by equation (A21). The derivative of the left-hand-side of equation (A21) with respect to $R^{k} / R$ is positive, implying that the threshold $p^{*}$ is increasing in $R^{k} / R$. Because the aggregate borrowing is given by (A26), it is increasing in $R^{k} / R$.

Next, the impact of $\underline{p}$ on the threshold $p^{*}$ and the aggregate borrowing $B$ is analyzed. With $F(p)$ given by a uniform distribution over $[\underline{p}, 1]$, the condition that determines $p^{*}$, (A21), is written as:

$$
\left(1-2 \frac{R}{R^{k}}\right) \frac{1-\phi}{1-2 \phi}\left[\left(\frac{p^{*}}{\underline{p}}\right)^{\frac{1-2 \phi}{1-\phi}}-1\right]=\frac{R}{R^{k}} \frac{1-\phi}{\phi}\left[\left(\frac{p^{*}}{\underline{p}}\right)^{-\frac{\phi}{1-\phi}}-1\right] .
$$

This equation implies that $p^{*} / \underline{p}$ is independent of $\underline{p}$. Hence, a drop in $\underline{p}$ decreases $p^{*}$, keeping $p^{*} / \underline{p}$ constant. In addition, $F\left(p^{*}\right)=\left(p^{*}-\underline{p}\right) /(1-\underline{p})=\left(p^{*} / \underline{p}-1\right) /(1 / \underline{p}-1)$ is increasing in $\underline{p}$. Hence, a drop in $\underline{p}$ decreases the aggregate borrowing (A26).

\section{B. General Equilibrium}

This section presents a system of equations for the models studied in the paper, which is followed by the calculation of steady state and the derivation of a key equation for lemons shocks. It also presents a numerical result on the real model with an exogenous markup in wages.

\section{B1. System of Equations}

In this section, a system of equations for the real model is presented, which is followed by equations pertaining to nominal wage rigidity for the nominal model.

A system of equations for the real model consists of the thirteen equations (B1)-(B13) with the same number of unknowns, $\left\{Y_{t}, C_{t}, I_{t}, K_{t}, h_{t}, N_{t}, u_{t}, p_{t}^{*}, \mathrm{CS}_{t}, r_{t}^{k}, w_{t}, q_{t}, R_{t}\right\}$.

Households: The optimality conditions for the household problem:

$$
\begin{aligned}
& 1=E_{t} \beta \frac{b_{t+1}}{b_{t}} \frac{C_{t}}{C_{t+1}} R_{t}, \\
& w_{t}=\psi h_{t}^{1 / \nu} C_{t} .
\end{aligned}
$$


Producers: The production function is given by:

$$
Y_{t}=\left(u_{t} K_{t-1}\right)^{\alpha} h_{t}^{1-\alpha}
$$

The optimality conditions for the consumption-good producer's problem:

$$
\begin{aligned}
& r_{t}^{k}=\alpha\left(u_{t} K_{t-1}\right)^{\alpha-1} h_{t}^{1-\alpha}, \\
& w_{t}=(1-\alpha)\left(u_{t} K_{t-1}\right)^{\alpha} h_{t}^{-\alpha},
\end{aligned}
$$

The law of motion for capital:

$$
K_{t}=(1-\delta) K_{t-1}+\left[1-S\left(\frac{I_{t}}{I_{t-1}}\right)\right] I_{t}
$$

The optimality condition for the capital-good producer's problem:

$$
1=q_{t} \mu_{t}\left[1-S\left(\frac{I_{t}}{I_{t-1}}\right)-S^{\prime}\left(\frac{I_{t}}{I_{t-1}}\right) \frac{I_{t}}{I_{t-1}}\right]+E_{t} \frac{\beta C_{t}}{C_{t+1}} \frac{b_{t+1}}{b_{t}} q_{t+1} \mu_{t+1} S^{\prime}\left(\frac{I_{t+1}}{I_{t}}\right)\left(\frac{I_{t+1}}{I_{t}}\right)^{2} .
$$

Entrepreneurs and banks: The capital utilization rate is given by:

$$
r_{t}^{k}=a^{\prime}\left(u_{t}\right)
$$

The balance sheet of the entrepreneurial sector is given by:

$$
q_{t} K_{t}=N_{t}+B_{t},
$$

where

$$
\begin{aligned}
B_{t} & =\frac{\phi\left(E_{t} R_{t+1}^{k} / R_{t}\right)}{1-\phi\left(E_{t} R_{t+1}^{k} / R_{t}\right)} F_{t}\left(p_{t}^{*}\right) N_{t}, \\
R_{t}^{k} & =\frac{r_{t}^{k} u_{t}+q_{t}(1-\delta)-a\left(u_{t}\right)}{q_{t-1}} .
\end{aligned}
$$

The law of motion for the aggregate net worth is given by:

$$
N_{t}=\gamma\left[R_{t}^{k}\left(N_{t-1}+B_{t-1}\right)-R_{t-1} B_{t-1}\right]+\xi Y_{t}
$$


The average credit spread is defined as:

$$
\begin{aligned}
\mathrm{CS}_{t} & =\frac{X_{t}}{B_{t}}-R_{t}=\frac{\frac{\phi E_{t} R_{t+1}^{k}}{1-\phi}\left(p_{t}^{*}\right)^{\frac{\phi}{1-\phi}} \int_{\underline{p}}^{p_{t}^{*}} p^{-\frac{1}{1-\phi}} d F_{t}(p)}{\frac{\phi\left(E_{t} R_{t+1}^{k} / R_{t}\right)}{1-\phi\left(E_{t} R_{t+1}^{k} / R_{t}\right)} F_{t}\left(p_{t}^{*}\right)}-R_{t} \\
& =\left(\frac{R_{t}-\phi E_{t} R_{t+1}^{k}}{1-\phi}\right) \frac{\left(p_{t}^{*}\right)^{\frac{\phi}{1-\phi}} \int_{\underline{p}}^{p_{t}^{*}} p^{-\frac{1}{1-\phi}} d F_{t}(p)}{F_{t}\left(p_{t}^{*}\right)}-R_{t}
\end{aligned}
$$

The banks' zero profit condition is given by:

$$
\int_{\underline{p}_{t}}^{p_{t}^{*}}\left[p f_{t}(p)-\frac{R_{t}}{E_{t} R_{t+1}^{k}} p f_{t}(p)-\frac{R_{t}}{E_{t} R_{t+1}^{k}} F_{t}(p)\right]\left(\frac{1}{p}\right)^{\frac{1}{1-\phi}} d p=0
$$

Market clearing: The market clearing condition for the consumption good is given by:

$$
Y_{t}=C_{t}+I_{t}+a\left(u_{t}\right) K_{t-1}
$$

The GDP is defined as $G D P_{t}=C_{t}+I_{t}$.

Nominal wage rigidity: A system of equations for the model with nominal wage rigidity is given by (B1)-(B13) with three modifications. First, equation (B2) is replaced by:

$$
w_{t}=\psi K_{w, t} / F_{w, t}
$$

where

$$
\begin{aligned}
& F_{w, t}=h_{t} / c_{t}+\beta \xi_{w} E_{t}\left[\left(w_{t} / w_{t+1}\right)^{\frac{\lambda w}{1-\lambda w}}\left(1 / \pi_{t+1}\right)^{\frac{1}{1-\lambda w}} F_{w, t+1}\right] \\
& K_{w, t}=h_{t}^{1+1 / \nu}+\beta \xi_{w} E_{t}\left[\left(w_{t} / w_{t+1}\right)^{\frac{\lambda w(1+1 / \nu)}{1-\lambda w}}\left(1 / \pi_{t+1}\right)^{\frac{\lambda w(1+1 / \nu)}{1-\lambda w}} K_{w, t+1}\right] .
\end{aligned}
$$

In these equations a subsidy is implicitly assumed so that equation (B14) is identical to (B2) in steady state. Second, the nominal bond is available for households so that the following Euler equations holds:

$$
1=E_{t} \beta \frac{C_{t}}{C_{t+1}} \frac{R_{t}^{n}}{\pi_{t+1}}
$$

where $R_{t}^{n}$ is the nominal interest rate and $\pi_{t+1}$ is the inflation rate. Third, the nominal 
interest rate follows a simple policy rule that depends only on current inflation:

$$
\log \left(R_{t}^{n} / R^{n}\right)=r_{\pi} \log \left(\pi_{t}\right),
$$

where $R^{n}$ is the nominal interest rate in steady state. To summarize, a system of equations for the model with nominal wage rigidity consists of (B1) and (B3)-(B16) with two additional unknowns $\left\{R_{t}^{n}, \pi_{t}\right\}$.

\section{B2. Steady State}

In calculating steady state, four target values are set for $L, C S, 1-F^{*}$, and $h$, where $L=q K / N$ is a leverage and $F^{*}=F\left(p^{*}\right)$. For the same number of parameters, $\phi, \underline{p}, \gamma$, and $\psi$, their values are pinned down along with steady state values for other endogenous variables. For normalization, $h$ is set as $h=1$.

From (B1), $R=1 / \beta$. Equation (B7) implies $q=1$. From (B8) and the assumed functional form of $a(\cdot), u=1$.

Fix $r^{k}$. From (B4), $K$ is given as $K=u^{-1}\left[r^{k} /\left(\alpha h^{1-\alpha}\right)\right]^{1 /(\alpha-1)}$. Output $Y$ is given by (B3) as $Y=(u K)^{\alpha} h^{1-\alpha}$. Wage $w$ is given by (B5) as $w=(1-\alpha)(u K)^{\alpha} h^{-\alpha}$. Investment $I$ is given by (B6) as $I=\delta K$. Consumption $C$ is given by (B13) as $C=Y-I-a(u) K$. Equation (B2) pins down parameter $\psi$ as $\psi=w /\left(h^{1 / \nu} C\right)$. Net worth $N$ is given as $N=q K / L$. Equation (B9) gives the loan as $B=q K-N$. Equation (B10) pins down parameter $\gamma$ as

$$
\gamma=\frac{N-\xi Y}{R^{k} N+\left(R^{k}-R\right) B},
$$

where $R^{k}=\left[r^{k} u+q(1-\delta)-a(u)\right] / q$. The assumption of $R^{k}-R>0$ ensures that $\gamma<1$. Equation (B9) pins down $\phi$ is given as

$$
\phi=\frac{R}{R^{k}} \frac{L-1}{F^{*}+L-1} .
$$

Because $F^{*}=\left(p^{*}-\underline{p}\right) /(1-\underline{p})$, threshold $p^{*}$ is given by $p^{*}=F^{*}(1-\underline{p})+\underline{p}$. Parameter $\underline{p}$ is pinned down by solving (B11) for $\underline{p}$ :

$$
C S=\frac{R-\phi R^{k}}{\phi} \frac{\left[\left(p^{*} / \underline{p}\right)^{\frac{\phi}{1-\phi}}-1\right]}{(1-\underline{p}) F^{*}}-R,
$$


where $p^{*}=F^{*}(1-\underline{p})+\underline{p}$. With $\underline{p}$ and $p^{*}$ in hand, adjust $r^{k}$ to satisfy (B12):

$$
0=\frac{1-\phi}{1-2 \phi}\left(1-\frac{2}{R^{k} / R}\right)\left[\left(p^{*}\right)^{\frac{1-2 \phi}{1-\phi}}-\underline{p}^{\frac{1-2 \phi}{1-\phi}}\right]-\frac{\underline{p}}{R^{k} / R} \frac{1-\phi}{\phi}\left[\left(p^{*}\right)^{-\frac{\phi}{1-\phi}}-\underline{p}^{-\frac{\phi}{1-\phi}}\right] .
$$

In the nominal model, $R^{n}=R$ and $\pi=1$ in steady state.

\section{B3. Derivation of Equation (28)}

Define $s_{t} \equiv E_{t} R_{t+1}^{k} / R_{t}$. Keeping in mind that $R^{e} / R^{f}$ and $F(p)$ in equation (10) in the paper correspond to $s_{t}$ and $F_{t}(p)=\left(p-\underline{p}_{t}\right) /\left(1-\underline{p}_{t}\right)$, respectively, log-linearizing equation (10) yields:

$$
\hat{p}_{t}^{*}=\chi_{3} \hat{s}_{t}+v_{t}
$$

with

$$
\chi_{3}=\frac{-\frac{1-\phi}{1-2 \phi}\left(\left(p^{*}\right)^{\frac{1-2 \phi}{1-\phi}}-\underline{p}^{\frac{1-2 \phi}{1-\phi}}\right)}{(1-2 / s)\left(p^{*}\right)^{\frac{1-2 \phi}{1-\phi}}+(\underline{p} / s)\left(p^{*}\right)^{-\frac{\phi}{1-\phi}}} .
$$

Because it is assumed that equation (10) in the paper has a unique solution, the comparative statistic analysis implies $\chi_{3}>0$. Next, log-linearizing equation (24) in the paper yields:

$$
\hat{s}_{t}=\frac{(1-\phi s) q K}{B}\left(\hat{q}_{t}+\hat{K}_{t}-\hat{N}_{t}\right)+(1-\phi s)\left(-\frac{p^{*}}{p^{*}-\underline{p}} \hat{p}_{t}^{*}+\frac{1-\bar{p}}{1-\underline{p}} \frac{\underline{p}}{p^{*}-\underline{p}} v_{t}\right) .
$$

Combining the above two log-linearized equations leads to equation (28):

$$
\hat{s}_{t}=-\chi_{1}\left(\hat{N}_{t}-\hat{q}_{t}-\hat{K}_{t}\right)-\chi_{2} v_{t}
$$

where

$$
\begin{aligned}
& \chi_{1}=\left\{1+\frac{(1-\phi s) p^{*}}{p^{*}-\underline{p}} \chi_{3}\right\}^{-1} \frac{(1-\phi s) q K}{B}>0, \\
& \chi_{2}=\left\{1+\frac{(1-\phi s) p^{*}}{p^{*}-\underline{p}} \chi_{3}\right\}^{-1} \frac{(1-\phi s)}{p^{*}-\underline{p}}\left(p^{*}-\underline{p} \frac{1-p^{*}}{1-\underline{p}}\right)>0 .
\end{aligned}
$$

\section{B4. Countercyclical Wage Markup}

In the paper, to overcome the co-movement problem between consumption and hours worked, nominal wage rigidity is introduced. As mentioned in the paper, however, what is important for addressing the problem is not nominal wage rigidity per se, but a counter- 
Figure S1: Impulse responses to a lemons shock
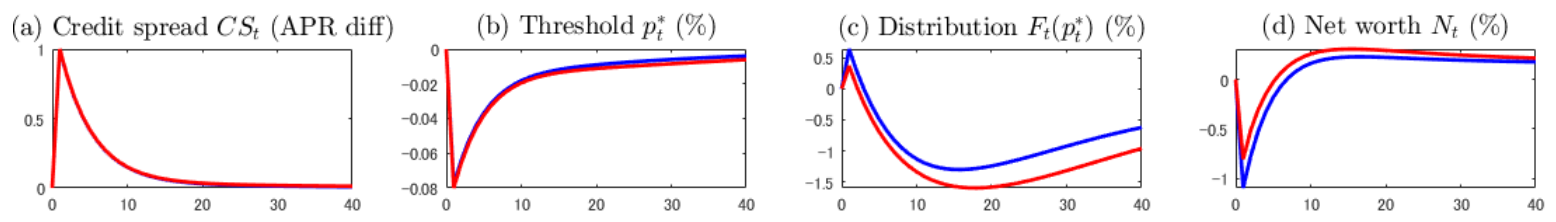

(e) Loans $B_{t}(\%)$

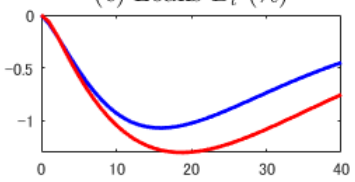

(f) Loans + net worth $B_{t}+N_{t}(\%)$

(g) Price of capital $q_{t}(\%)$
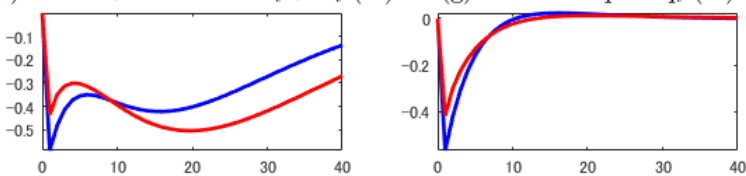

(h) Investment $I_{t}(\%)$

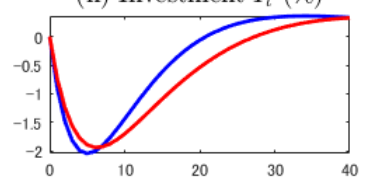

(i) Output $Y_{t}(\%)$

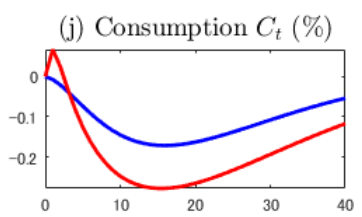

(k) Hours worked $h_{t}(\%)$

(1) Excess return $\frac{E_{t} R_{t+1}^{k}}{R_{t}}($ APR diff
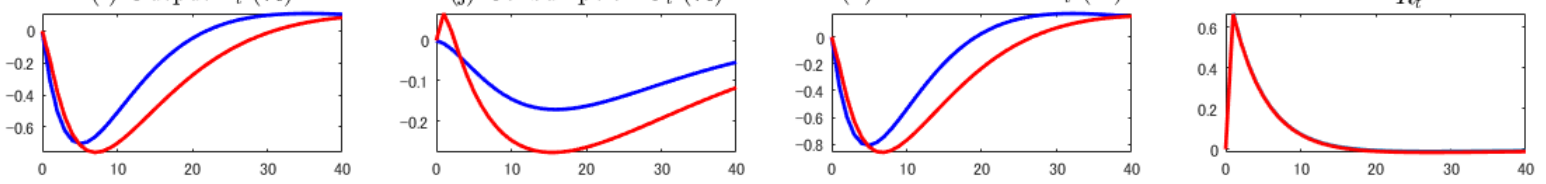

—Real model with exo. markup —Full model

cyclical markup in wages. To see it clearly, a model, which is identical to the full model in the paper but with nominal wage rigidity replaced by an exogenous countercyclical markup in wages, is studied. In particular, such a model is the real model, presented in Section B1, with equation (B2) replaced by:

$$
w_{t}=\lambda_{w, t} \psi h_{t}^{1 / \nu} C_{t}
$$

where $\lambda_{w, t}$ is an exogenous countercyclical markup in wages, given by $\lambda_{w, t}=\left(Y_{t} / Y\right)^{-\omega}$. In steady state, equations (B2) and (B20) coincide, but in dynamics the markup fluctuates countercyclically, where the degree of countercylicality is governed by parameter $\omega>0$.

Figure S1 plots impulse responses to a persistent lemons shock with its AR(1) coefficient given by $\rho_{e}=0.8$ for the full model (red lines) and the real model with an exogenous markup in wages (blue lines), where $\omega$ is set as $\omega=1.4$. The value of $\omega$ is not too high, given that a wage markup is about twice as volatile as output as reported by Gali, Gertler, and Lopez-Salido (2007). Overall, the two models show similar responses to the lemons shock. Importantly, consumption does not increase but decrease gradually for the model with an exogenous markup in wages. Figure S1 confirms that the co-movement problem can be overcome even in the real model if a markup in wages moves countercyclically enough. 


\section{Other Models}

\section{C1. Risk-Shock Model}

A risk-shock model is the full model that replaces the four equations pertaining to financial frictions, (B9)-(B12), and the good-market clearing condition, (B13), with the following equations:

$$
\begin{aligned}
& q_{t} K_{t}=L_{t} N_{t} \\
& 0=E_{t}\left[\left[1-\Gamma_{t+1}\left(\bar{\omega}_{t+1}\right)\right] s_{t+1}+\frac{\Gamma_{t+1}^{\prime}\left(\bar{\omega}_{t+1}\right)\left\{\left[\Gamma_{t+1}\left(\bar{\omega}_{t+1}\right)-\mu G_{t+1}\left(\bar{\omega}_{t+1}\right)\right] s_{t+1}-1\right\}}{\Gamma_{t+1}^{\prime}\left(\bar{\omega}_{t+1}\right)-\mu G_{t+1}^{\prime}\left(\bar{\omega}_{t+1}\right)}\right], \\
& 0=\left[\Gamma_{t+1}\left(\bar{\omega}_{t+1}\right)-\mu G_{t+1}\left(\bar{\omega}_{t+1}\right)\right] s_{t+1} L_{t}-L_{t}+1, \\
& N_{t}=\gamma\left[1-\Gamma_{t}\left(\bar{\omega}_{t}\right)\right] R_{t}^{k} L_{t-1} N_{t-1}+\xi Y_{t}, \\
& \mathrm{CS}_{t}=E_{t} \frac{\bar{\omega}_{t+1} R_{t+1}^{k} L_{t}}{L_{t}-1}-R_{t},
\end{aligned}
$$

where

$$
\begin{aligned}
& G_{t+1}(\bar{\omega})=\int_{0}^{\bar{\omega}} \omega d F_{t+1}^{r}(\omega), \\
& \Gamma_{t+1}(\bar{\omega})=\left[1-F_{t+1}^{r}(\bar{\omega})\right] \bar{\omega}+G_{t+1}(\bar{\omega}), \\
& s_{t}=E_{t} R_{t+1}^{k} / R_{t},
\end{aligned}
$$

and $F_{t}^{r}$ denotes a log-normal distribution with mean zero: $F_{t}^{r}=\log \mathcal{N}\left(-\sigma_{t}^{2} / 2, \sigma_{t}^{2}\right)$. The standard deviation of $F_{t}^{r}$ is time varying and is given by $\sigma_{t}=\sigma_{0} e^{v_{t}^{r}}$, where $v_{t}^{r}$ is a risk shock. Equation (C1) defines a leverage $L_{t}$ and equation (C2) summarizes entrepreneurs' optimal choice of contract, where $\bar{\omega}_{t}$ is a threshold of an idiosyncratic shock to entrepreneurs. If a realized idiosyncratic shock is less than $\bar{\omega}_{t}$, entrepreneurs go bankrupt. Equation (C3) is a zero-profit condition of banks, equation (C4) is the law of motion for the net worth, and equation (C5) defines the credit spread. For the detail of the derivation of these equations, see CMR (2014) or Christiano and Ikeda (2013, Section 6).

Regarding parameter values, the monitoring cost, $\mu$, is set at 0.2 , which is the lower bound of the range of 0.2 to 0.36 that Carlstrom and Fuerst (1997) argued as empirically relevant. The standard deviation in steady state, $\sigma_{0}$, and the surviving rate, $\gamma$, are jointly set to hit the same targets as in the full model: $q K / N=2$ and $\mathrm{CS}=1.88$. The results presented in the main text are robust to the value of $\mu$ within its range of 0.2 to 0.36 . 


\section{C2. Shocks to Lenders' Perception of Borrowers' Quality}

Let $\eta_{t}$ denote a perception shock. A threshold, $p_{t}^{*}$, is determined by equation (B12) with $\underline{p}_{t}$ given by $\underline{p}_{t}=\underline{p} e^{v_{t}+\eta_{t}}$, where $v_{t}$ is a lemons shock. The aggregate loan $B_{t}$ is given by the integral of an individual bank loan,

$$
B_{n, t}(p)=\left[\frac{1}{1-\phi}\left(\frac{p_{t}^{*}}{p}\right)^{\frac{\phi}{1-\phi}}-1\right] N_{n, t}
$$

over the range of $\left[\underline{p} e^{v_{t}}, p_{t}^{*}\right]$ and over individual net worth:

$$
\begin{aligned}
B_{t} & =\frac{N_{t}}{1-\underline{p} e^{v_{t}}} \int_{\underline{p} e^{v_{t}}}^{p_{t}^{*}}\left[\frac{1}{1-\phi}\left(\frac{p_{t}^{*}}{p}\right)^{\frac{\phi}{1-\phi}}-1\right] d p, \\
& =\left[\frac{1}{1-2 \phi} \frac{\left(p_{t}^{*}\right)^{\frac{\phi}{1-\phi}}}{1-\underline{p} e^{v_{t}}}\left(\left(p_{t}^{*}\right)^{\frac{1-2 \phi}{1-\phi}}-\left(\underline{p} e^{v_{t}}\right)^{\frac{1-2 \phi}{1-\phi}}\right)-\frac{p_{t}^{*}-\underline{p} e^{v_{t}}}{1-\underline{p} e^{v_{t}}}\right] N_{t} .
\end{aligned}
$$

The aggregate repayment $X_{t}$ under banks' perception of borrowers' riskiness is given by the integral of an individual bank repayment,

$$
X_{n, t}(p)=\left[\frac{\phi E_{t} R_{t+1}^{k}}{1-\phi}\left(p_{t}^{*}\right)^{\frac{\phi}{1-\phi}}\right]\left(\frac{1}{p}\right)^{\frac{1}{1-\phi}} N_{n, t}
$$

over the range of $\left[\underline{p} e^{v_{t}}, p_{t}^{*}\right]$ and over individual net worth:

$$
\begin{aligned}
X_{t} & =N_{t}\left[\frac{\phi E_{t} R_{t+1}^{k}}{1-\phi} \frac{\left(p_{t}^{*}\right)^{\frac{\phi}{1-\phi}}}{1-\underline{p} e^{v_{t}}}\right] \int_{\underline{p} e^{v_{t}}}^{p_{t}^{*}} p^{-\frac{1}{1-\phi}} d p, \\
& \left.=\frac{E_{t} R_{t+1}^{k}\left(p_{t}^{*}\right)^{\frac{\phi}{1-\phi}}}{1-\underline{p} e^{v_{t}}}\left(\underline{p} e^{v_{t}}\right)^{-\frac{\phi}{1-\phi}}-\left(p_{t}^{*}\right)^{-\frac{\phi}{1-\phi}}\right) N_{t} .
\end{aligned}
$$

The credit spread is given by $\mathrm{CS}_{t}=X_{t} / B_{t}$, where $B_{t}$ and $X_{t}$ are given by $(\mathrm{C} 6)$ and $(\mathrm{C} 7)$ respectively. The actual aggregate repayment $\tilde{X}_{t}$ received by banks ex-post is given by

$$
\begin{aligned}
\tilde{X}_{t}=\int_{\underline{p} e^{v_{t}}}^{p_{t}^{*}} \frac{p X_{n, t}(p)}{1-\underline{p} e^{v_{t}}} d p & =N_{t}\left[\frac{\phi E_{t} R_{t+1}^{k}}{1-\phi} \frac{\left(p_{t}^{*}\right)^{\frac{\phi}{1-\phi}}}{1-\underline{p} e^{v_{t}}}\right] \int_{\underline{p} e^{v_{t}}}^{p_{t}^{*}} p^{1-\frac{1}{1-\phi}} d p, \\
& =\left[\frac{\phi E_{t} R_{t+1}^{k}}{1-2 \phi} \frac{\left(p_{t}^{*}\right)^{\frac{\phi}{1-\phi}}}{1-\underline{p} e^{v_{t}}}\right]\left(\left(p_{t}^{*}\right)^{\frac{1-2 \phi}{1-\phi}}-\left(\underline{p} e^{v_{t}}\right)^{\frac{1-2 \phi}{1-\phi}}\right) N_{t} .
\end{aligned}
$$


With a perception shock, the law of motion for the net worth (B10) is replaced by:

$$
N_{t}=\gamma\left[R_{t}^{k}\left(N_{t-1}+B_{t-1}\right)-\tilde{X}_{t-1}\right]+\xi Y_{t}
$$

where $B_{t}$ and $\tilde{X}_{t}$ are given by (C6) and (C8), respectively.

\section{References}

[1] Gali Jordi, Mark Gertler, and J. David Lopez-Salido (2007), "Markups, Gaps, and the Welfare Costs of Business Fluctuations," The Review of Economics and Statistics, 89(1), 44-59. 